\title{
Recovering Aerodynamic Side Loads on Rocket Nozzles using Quasi-Static Strain-Gage Measurements
}

\author{
Andrew M. Brown ${ }^{1}$, Joseph H. Ruf ${ }^{2}$, David M. McDaniels ${ }^{3}$ \\ NASA Marshall Space Flight Center, Marshall Space Flight Center, Alabama 35812
}

\begin{abstract}
During over-expanded operation of rocket nozzles, which is defined to be when the exit pressure is greater than internal pressure over some part of the nozzle, the nozzle will experience a transverse forcing function due to the pressure differential across the nozzle wall. Overexpansion occurs during the nozzle start-up and shutdown transient, even in high-altitude engines, because most test facilities cannot completely reproduce the nearvacuum pressures at those altitudes. During this transient, the pressure differential moves axially down the nozzle as it becomes pressurized, but this differential is never perfectly symmetric circumferentially. The character of the forcing function is highly complex and defined by a series of restricted and free shock separations. The subject of this paper is the determination of the magnitude of this loading during sub-scale testing via measurement of the structural dynamic response of the nozzle and its support structure. An initial attempt at back-calculating this load using the inverse of the transfer function was performed, but this attempt was shown to be highly susceptible to numerical error. The final method chosen was to use statically calibrated strain data and to filter out the system fundamental frequency such that the measured response yields close to the correct dynamic loading function. This method was shown to capture $93 \%$ of the pressure spectral energy using controlled load shaker testing. This method is one of the only practical ways for the inverse determination of the forcing function for non-stationary excitations, and, to the authors' knowledge, has not been described in the literature to date.
\end{abstract}

\section{Nomenclature}

$\begin{array}{ll}\text { CFD } & =\text { Computational Fluid Dynamics } \\ \text { FSS } & =\text { Free Shock Separation } \\ \text { NPR } & =\text { Nozzle Pressure Ratio } \\ \text { NTF } & \text { MSFC Nozzle Test Facility } \\ \mathrm{P}_{\mathrm{w}} & =\text { Local Nozzle Wall Pressure } \\ \mathrm{P}_{\mathrm{amb}} & =\text { Ambient External Pressure } \\ \text { PAR } & =\text { Parabolic Contour } \\ \text { PSD } & =\text { Power Spectral Density } \\ \text { RSS } & =\text { Restricted Shock Separation } \\ \text { SDOF } & =\text { Single Degree of Freedom } \\ \text { SSME } & =\text { Space Shuttle Main Engine } \\ \text { SSPM } & =\text { Skewed Shock Plane Method } \\ \text { TIC } & =\text { Truncated Ideal Contour } \\ \text { TOC } & =\text { Thrust Optimized Contour }\end{array}$

\footnotetext{
${ }^{1}$ Lead Aerospace Engineer, ER41/Propulsion Structural \& Dynamics Analysis Branch, AIAA Senior Member.

${ }^{2}$ Aerospace Engineer, ER42/Propulsion Delivery Fluids Branch, AIAA Member

${ }^{3}$ Aerospace Engineer, ER42/Propulsion Delivery Fluids Branch
} 


\section{Introduction}

During over-expanded operation of rocket nozzles, which is defined to be when the exit pressure is greater than internal pressure over some part of the nozzle, the nozzle will experience a radially inward forcing function due to the pressure differential across the nozzle wall. Overexpansion occurs during nozzle start-up and shutdown transient, and even occurs in nozzles for high-altitude engines because most test facilities cannot completely reproduce the near-vacuum pressures of those altitudes. During the start-up transient, the pressure differential moves axially down (or up in shutdown) the nozzle as it becomes pressurized, but this differential is never perfectly symmetric circumferentially. The radial force resolves, therefore, as a transverse load, which has been termed in the industry as a "side load." These side loads are of a large enough magnitude to cause failure of engines during testing and is a major driver in the design of rocket engines.

The character of the forcing function is highly complex; an excellent survey of the literature in the subject is provided by Ostlund ${ }^{1}$. The dominating fluid dynamics are the Free Shock Separation (FSS) and the Restricted Shock Separation (RSS) and the transition between the two flow structures. The FSS occurs when the developing nozzle flow in the diverging section suddenly separates from the wall; since this is frequently non-axisymmetric, it will induce a "side load". The parameter driving the axial location of the FSS is the wall pressure, $p_{w}$, relative to the ambient pressure, $p_{a m b}$. Experimental and numerical data indicate FSS occurs when the local $p_{w}$ decreases to a value of about $30 \%$ of $p_{\text {amb }}$. In RSS the separated flow reattaches to the wall; again, this is usually an asymmetric event. Mach contours from computational fluid dynamic (CFD) simulations of each of these cases are shown in Fig.1. Different types of nozzle contours lead to a predominance of each of the events. Thrust optimized contours (TOC), such as that used in the Space Shuttle Main Engine (SSME), can induce RSS which leads to the large side loads and violent response clearly seen in high-speed video of the SSME during its start-up. The Truncated Ideal Contour (TIC), which has not been used for rocket nozzles in quantity, is predicted numerically to not have RSS and, therefore should exhibit lower side loads.

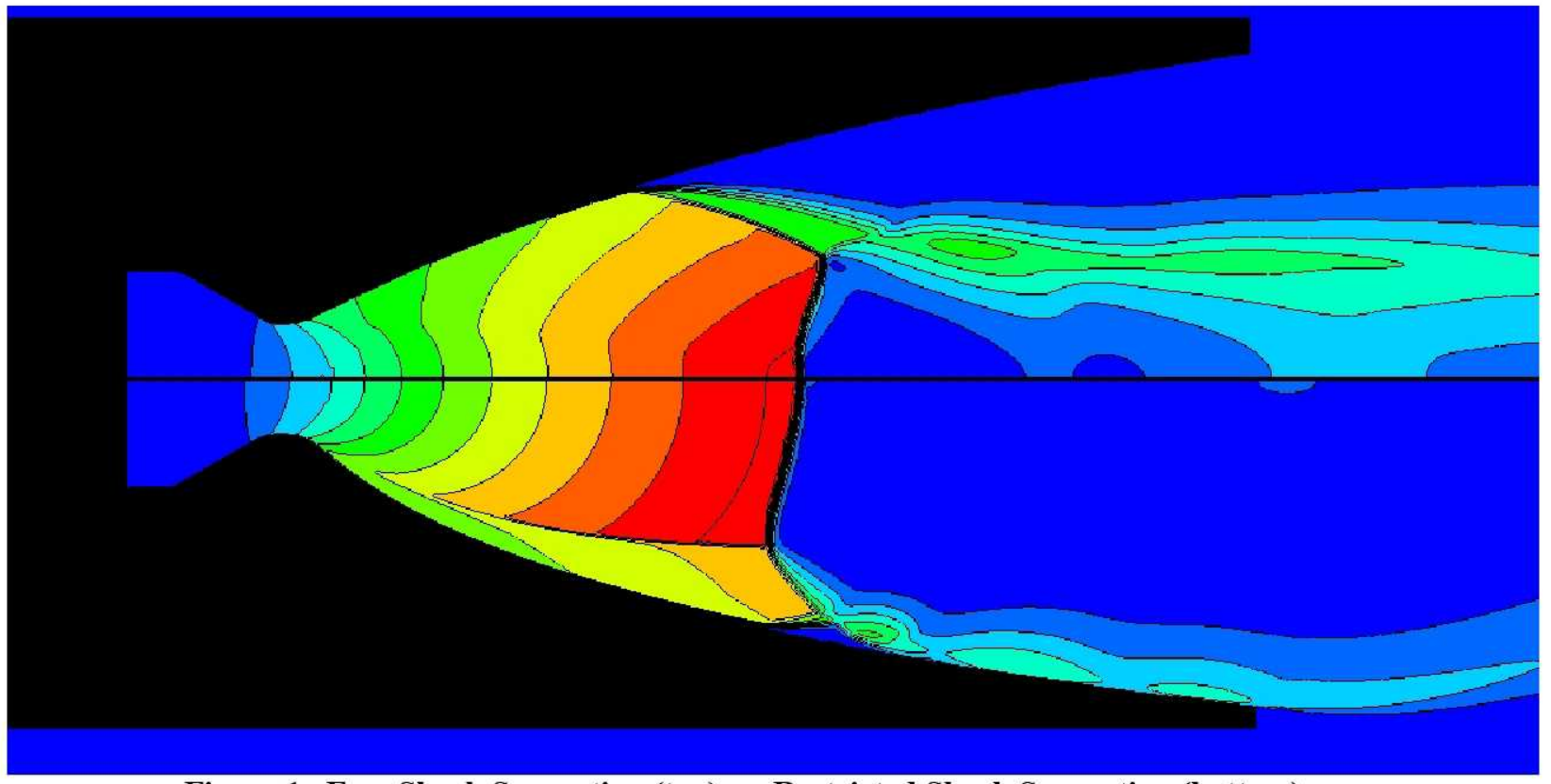

Figure 1. Free Shock Separation (top) vs. Restricted Shock Separation (bottom)

Because side loads can be of large magnitude, the authors have undertaken a multi-year research effort into understanding the phenomena with the goal of designing more efficient rocket engine nozzle structures. ${ }^{2,3}$ The most recent phase has concentrated on characterizing the side load reduction by changing from the traditional thrust optimized contours to a TIC. The motivation lies not only in understanding the basic physics and providing a foundation for future nozzle designs, but specifically for refining design load sets for the J2-X engine that will power the upper stage of the new NASA Ares I and Ares V launch vehicles. This project is based on experimental measurements on sub-scale nozzles during cold-flow start simulations at the NASA/Marshall Space Flight Center 
(MSFC) Nozzle Test Facility (NTF). A wide array of instrumentation including strain gages, accelerometers, and static and high frequency Kulite pressure gages were applied to quantify both the character and magnitude of the aerodynamic forcing function on the sub-scale nozzles (see Fig. 2). This value can be then empirically tied to the basic nozzle performance design parameters (contour, chamber pressure, expansion ratio) and can also be used to validate CFD analysis of these systems. The focus of this paper is on the methodologies attempted and finally used to characterize the magnitude of the aerodynamic forces acting on the sub-scale nozzles at the MSFC NTF.

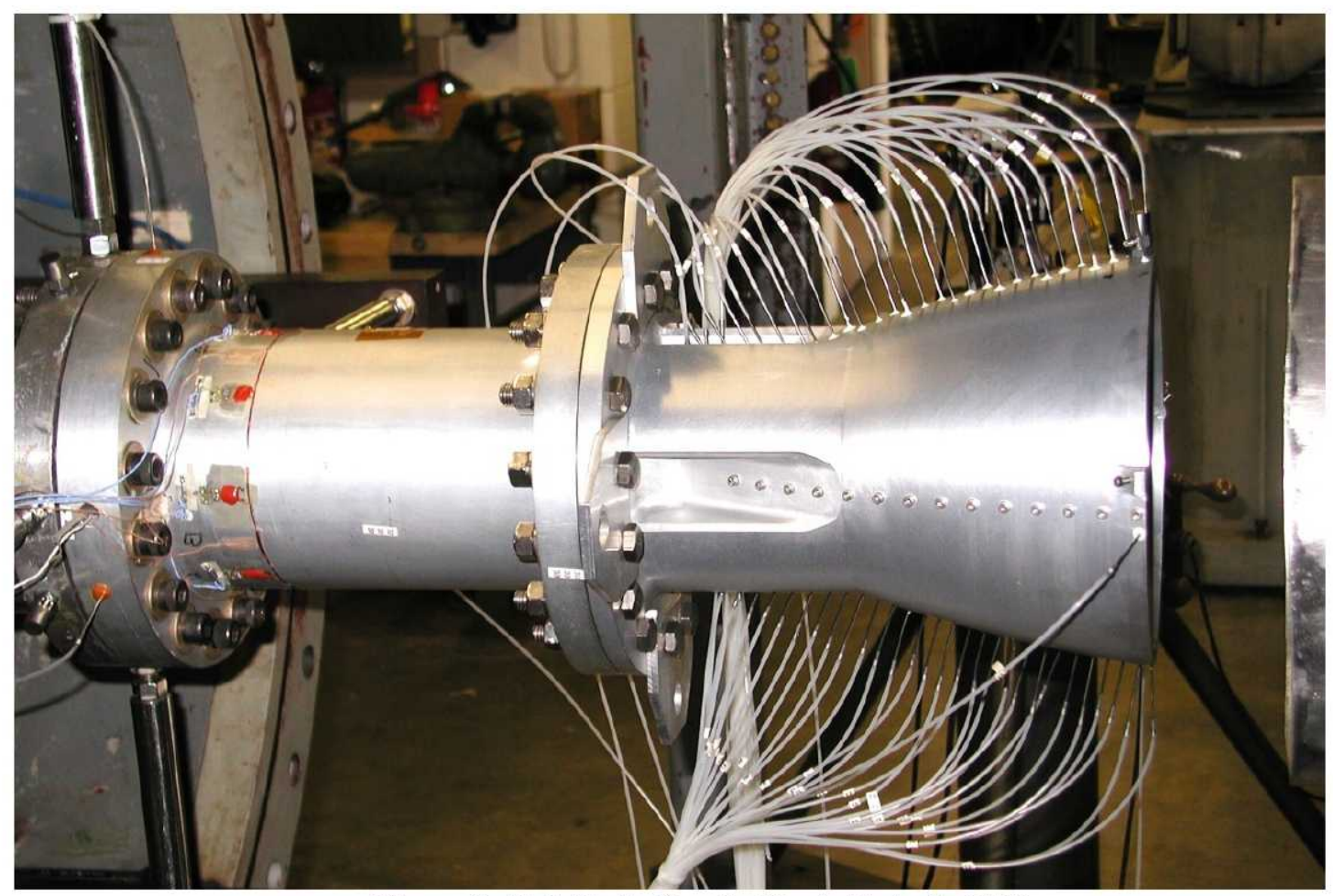

Figure 2. Sub-Scale Nozzle, Strain Tube in NTF

\section{Calculation of Magnitude of Side Load}

There has been substantial research into the characterization of the fluid dynamics of side loads as a function of engine and nozzle parameters. However, there has not been sufficient data available domestically for deriving a reliable value of the magnitude of this loading for flight hardware. This is in general both a difficult theoretical and experimental problem; the only true measure of the forcing function is pressure gage data, and it is impractical to provide a dense enough array to accurately measure the load. This situation therefore leaves measurement of nozzle and engine response as the primary tool to obtain the forcing function, as well as an accurate representation of the stiffness, damping, and inertial quantities of the engine system.

The current standard domestic methodology is the "Skewed-Shock Plane Method (SSPM)," which was developed during the development of the J2-S rocket engine during the 1970's. ${ }^{4}$ This method assumes that a skewed plane describing the separated pressure front exists along the wall starting at one circumferential location where $p_{w}$ equals half of $p_{a m b}$ and extending to the opposite side of the nozzle where the ratio between the two is 0.25 (Fig. 3). This method, which is a gross description of the FSS flow structure, was not intended to represent the true flow structure (especially the RSS), but was empirically generated to envelope the engine structural dynamic response when the transverse loading resulting from the calculated force was applied statically. The SSPM is the baseline method for generating the $\mathrm{J} 2$-X nozzle side load design load set.

Since the SSPM method was developed based on the J2-S side loads and later updated with side load measurements from the SSME, it is believed to over-predict the side loads that would be generated by a TIC nozzle. Experimental data was therefore needed to assess the degree to which the SSPM over-predicted these TIC side loads. This motivated the initiation of a test program using the existing Nozzle Test Facility at MSFC, which was 
modified to enable measurement of off-axis forces. This facility uses sub-scale nozzles, for which the side loads are substantially easier to characterize than for full-scale nozzles.

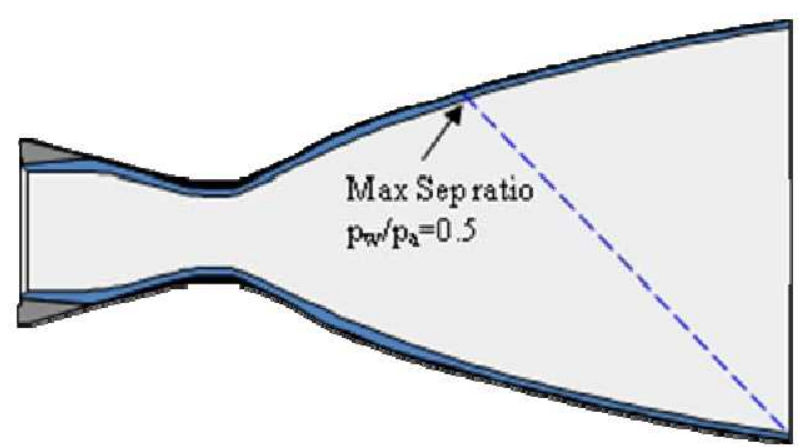

Figure 3. Skewed Shock Plane Method

The experimental approach for the test program was based on the work published by Frey \& Stark in $2000 .^{5}$ The nozzle test article is mounted on a flexible, easy-to-characterize "strain tube" which uses strain gages to measure the system response. The nozzle test article itself is very stiff, with a fundamental frequency well above the expected excitation frequency range of $0-300 \mathrm{~Hz}$. Several procedures for recovering the aerodynamic forcing function on this system were attempted and will now be discussed.

\section{Initial Attempts of Inverse Force determination}

Following the Frey \& Stark procedure, the first step in the attempt to recover the forcing function was to generate a complex frequency response of the experimental system by creating a finite element model and performing a modal test on the experimental system to verify it. Because of the poor definition of the test facility, the finite element model was replaced by a modal test-generated "pseudo-finite element model" of the system, which uses multi-point constraint equations tying together generalized modal coordinates, represented by NASTRAN "spoints." Acceleration transfer functions for acceleration for a forcing function assumed at a single location were taken directly from the modal test. For a SDOF system, if the responses are then divided by the square of the transfer function, the RMS of the result is the input force divided by the mass (if it is assumed to be applied at a single location). Details on the development of this technique were reported by the authors in 2007. ${ }^{6}$ Although there were some errors, especially in the region of the second flexible mode, the technique was deemed accurate enough to carry on to the application phase, which required automation to calculate side loads from hundreds of different cold-flow test runs. An automated process to perform the acceleration-transfer function squared division was implemented in the data analysis software package PC-Signal (an example is shown in Fig. 4), but unfortunately, the results were not consistent for different choices of block size and appeared to be unrealistically skewed in regions away from the fundamental structural frequency. These errors reflect the susceptibility of this method to numerical error in regions of low transfer function values, a problem discussed in detail by Kammer in $1996^{7}$. The high sensitivity to sample block size was probably due to the fact that the excitation of the system is non-stationary.

A second method pursued was to represent the entire system by a two degree of freedom (tip displacement and rotation) model with explicit mass, stiffness, and damping values, multiply these by the measured acceleration and derived values of the velocity (from acceleration) and deflection (from strain) to calculate a force. An Euler beam assumption was used to model the strain tube and the nozzle was modeled as a rigid mass on a rigid beam beyond the node point (see Fig. 5). One problematic issue was identifying the rotational stiffness and inertia. This was accomplished by estimating initial values for these parameters based upon the geometry, solving for the natural frequencies, comparing these with the measured values from modal test, and iterating until agreement was obtained. A static displacement test, loading the structure horizontally and vertically through a single point and measuring the displacement with both dial gages and photogrammetry was attempted to verify the estimate for the stiffness parameter. However, neither measurement technique proved sufficiently accurate for the small deflection in this stiff 
system. A shaker test was then performed to try to verify the dynamic response of the finite element model. The experimental system was excited with a known wide band random input force (both horizontally and vertically) and strains and accelerations were measured at the same locations as during the cold-flow testing. The shaker test displacements were then applied to the two-degree-of-freedom model, and the recovered forces were compared with the measured forces applied during the shaker test. Initial indications comparing results for the same test were positive, but when these system parameters were used for a different shaker test run, the error was greater than $50 \%$. This unacceptable error is probably due to the difficulty in deriving and/or measuring the rotational displacements, velocities, and accelerations as well as deriving the translational displacement from the strain measurement of the experimental hardware.
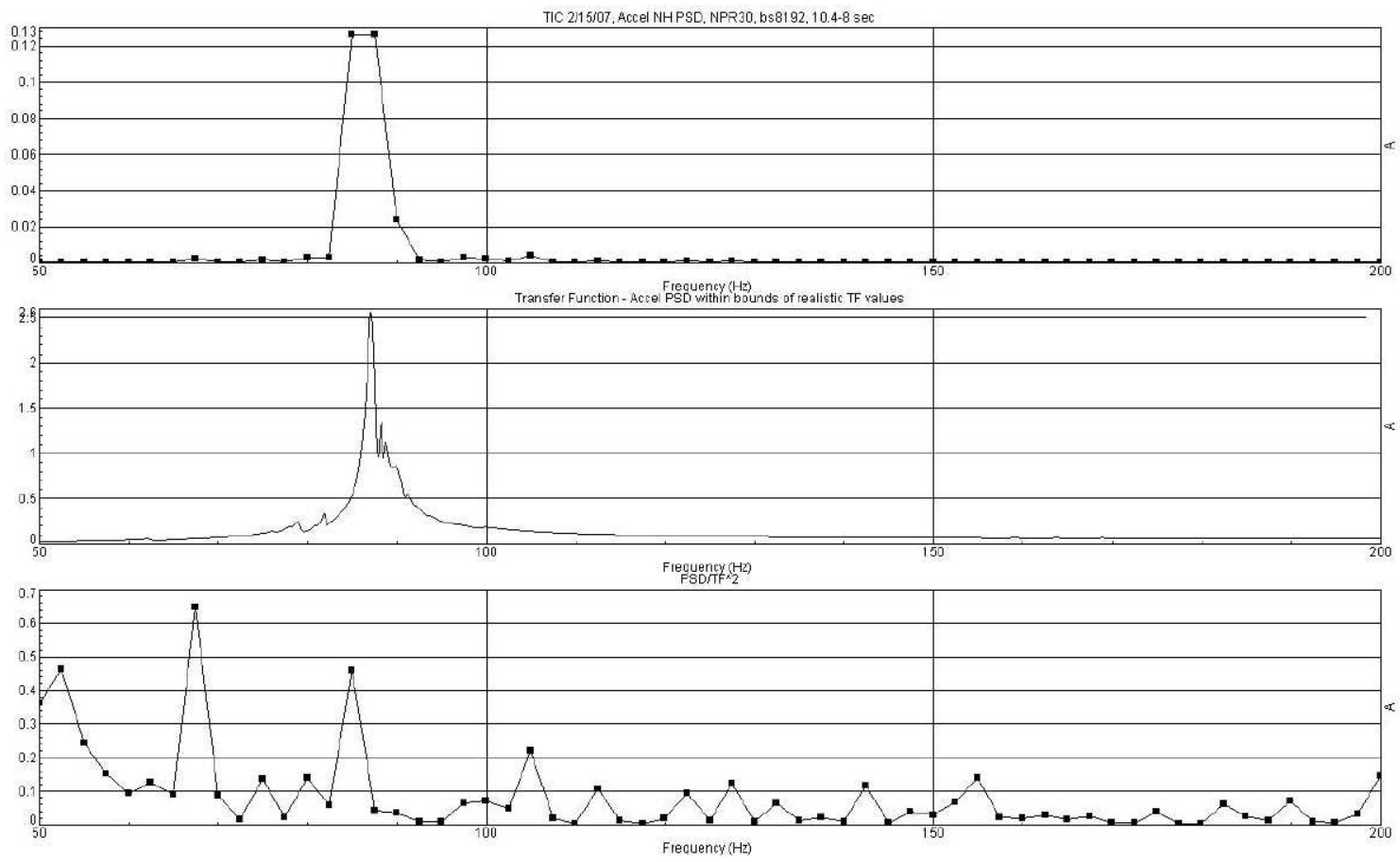

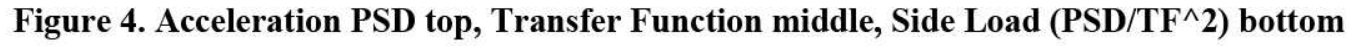
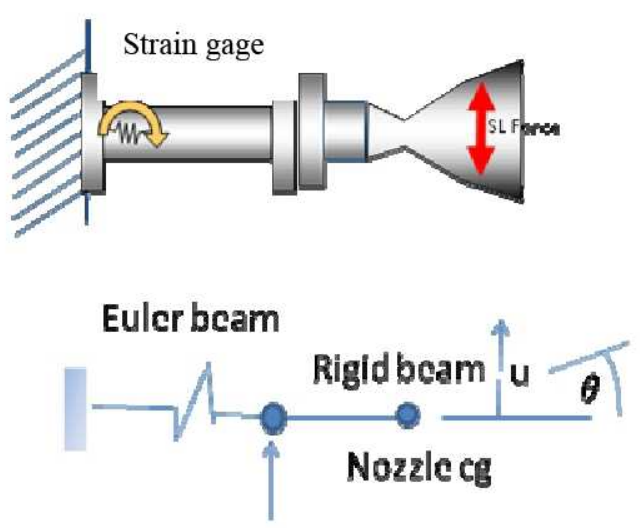

$$
\begin{aligned}
& \text { Assumed Forec } \\
& \text { application } \\
& \text { point }
\end{aligned}
$$

Figure 5. Two DOF model of System 


\section{Measurement of Quasi-Static Forces}

Although the methods described above were considered to be the primary method for obtaining the aerodynamic side load, a rigorous procedure for obtaining "quasi-static" side loads was also being undertaken in parallel. The purpose of this procedure was to obtain a rapidly observable value that could guide testing in real-time and make gross assessments. A converging nozzle with no flow-induced off-axis loads was used for the calibration process. This nozzle had a provision for hanging weight in both off-axis planes. Temperature was carefully maintained in the strain tube wall by flowing a small amount of temperature controlled air through this calibration nozzle during the weight hanging process. Calibration weights were hung via a system of knife edges and pulleys designed such that pure horizontal and vertical static loads could be applied to the nozzle in combination. A full factorial experimental design was used, providing every combination of weights in a 2 factor, 5 level matrix. Strain gages were arranged on the strain tube in a full-bridge arrangement to minimize thermal strain errors, with active elements in both tension and compression, at two different moment arms from the load application point. The static load in each direction as a function of the gage readings in each axis was then calculated as a response surface using multiple linear regressions of this static calibration data. These calibration equations are dependent on temperature effects, as well as amplifier gain settings necessary. Each significantly different set of test conditions required a different calibration. The set of equations for the most frequently tested configuration are shown below.

Moment wartcal $=21.9619+$ gtraln gago wartical $* 36.6987+$ gtrain gage horizontal $*-1.42623$

$$
\text { Moment horizontal }=31.2856+\text { strain gage vertical } * 1.0829+\text { strain gage horizontal } * 37.8256
$$

These equations were implemented into the data acquisition software so a real time "side-load" could be observed during testing. However, early modal testing of the experimental setup showed it had a fundamental system resonance of $87 \mathrm{~Hz}$, well within the range of the expected excitation spectrum of the aerodynamic forces. Therefore, this "quasi-static" force would obviously not reflect the true dynamic aerodynamic force, since the wideband excitation would be dominated by the resonant frequency.

After the failure of the inverse frequency response and dynamic replication methods described in the previous section, though, the authors realized that these "quasi-static" values could provide the design parameters sought after. The first step was to filter out the strain gage signal in the region around the system's fundamental frequency. This action leaves only that portion of the response that is not magnified. If the remaining portion of the excitation spectrum is assumed to be relatively similar for the two types of nozzle contours under consideration, then a ratio between the two quasi-static response measurements can be calculated and applied as a "knock-down" factor on the side load design load set. If the data in region around the resonance were not removed, then this ratio would be incorrectly dominated by that region; the filtering provides more confidence that an accurate overall ratio of the total energy of the excitation spectra is obtained.

Although this methodology was felt to be reasonable, the location of the fundamental frequency in the center of the highest-energy region of the excitation spectra was not desirable because a non-trivial portion of this spectrum would be removed from the ratio calculation. A design effort was therefore undertaken to stiffen the structure to increase the fundamental frequency. After several design iterations, a solution was found that moved the natural frequency up to $188.5 \mathrm{~Hz}$.

The size of the "notch" filter applied was arrived at initially by examining the response power spectral densities (PSD's). A value of $\pm 22.5 \mathrm{~Hz}$ around the resonant peak was selected based upon the point at which the response appeared to be magnified by the resonance. This value was verified by looking at magnification plots obtained later (discussed in shaker test section below). After some iteration on filter effectiveness, other chosen filter parameters are a Hamming filter type, filter order of 1023, and block size of 4096. Figure 6 shows the desired effect of implementing this $45 \mathrm{~Hz}$-wide filter centered around the fundamental system frequency to remove the magnification due to the resonance.

Both the filtered and unfiltered strain gage data was imported into Matlab for application of the calibration equations, statistical assessment, and plotting. Because the critical parameter for side loads is the nozzle pressure ratio (NPR), the information necessary to calculate this value was also imported. Since the NPR varied up and down during the testing, the side load statistics were collected in NPR bins of size 5. The maximum values across the test series for each NPR bin were then collated for each nozzle contour and are compared to get the desired knockdown 
factor, as shown in Fig. 7. More details on the methodology used to generate the knockdown factor, including facility correction factors and uncertainty quantification, will be published by the authors in a future paper.

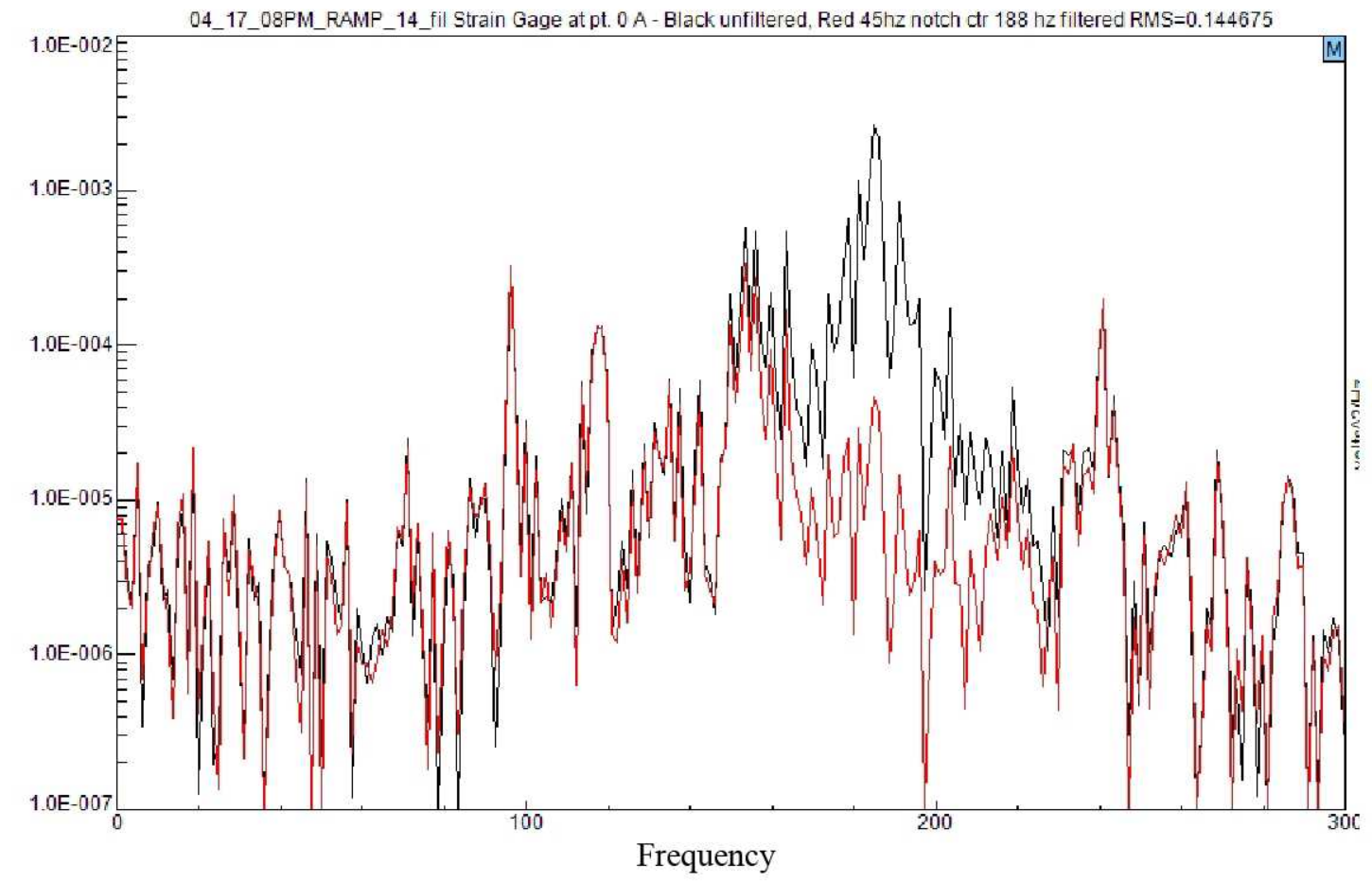

Figure 6. Comparison of Filtered (red) with Unfiltered (black) Strain Gage PSD Measurements

\section{Shaker Test}

A side benefit of the shaker test performed to support the attempted TDOF dynamic methodology was that both the measured input force and the "quasi-static" force could be recovered. First, this allowed the generation of the magnification function:

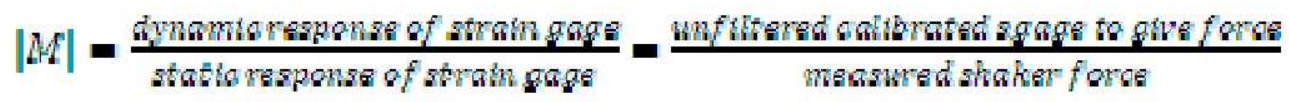

The resulting function is shown in Fig. 8. It was used to validate the selection of the $45 \mathrm{~Hz}$ filter centered on the resonance used in the side load calculations. The flat region well below the natural frequency has been normalized, since there is no magnification in the very low frequency range of a dynamic system, and this yields a $|\mathrm{M}| \approx 1.3$ at the low end of the filter band of $166 \mathrm{~Hz}$ and the same value at the high end of the filter band of $211 \mathrm{~Hz}$. This verifies that the $45 \mathrm{~Hz}$ filter is appropriate for capturing the unmagnified portion of the excitation spectrum.

Secondly, the shaker test data was used to compare the time history of the measured shaker force with the force obtained using the calibration equations for both the unfiltered and the filtered strain gage measurements. A sample of this comparison is shown in Fig. 9. As expected, the unfiltered stain gage derived force significantly exceeds the actual force and is dominated by the fundamental system frequency which magnifies the response and acts as a mechanical band-pass filter at that frequency. The filtered force, on the other hand, is reasonably close to the actual force applied by the shaker, both in frequency character and in magnitude.

The quantitative assessments of these comparisons, which were obtained by looking at the statistics of the time histories (see the title in Fig. 9), show a much larger error between the unfiltered measurement and the actual force than the filtered measurement and the actual force. This validates that the filtering procedure resulted in a much 
more accurate value for the forcing function. The filtered three-sigma force is about $33 \%$ below the actual force, more than would be expected if the excitation force was white noise between $0-300 \mathrm{~Hz}$, for which the filtering process would remove just $15 \%$ of the energy $(45 \mathrm{~Hz}$ out of $300 \mathrm{~Hz})$. However, due to shaker controller design, the shaker excitation spectrum is not representative of the expected aerodynamic spectrum, in particular in the region just above the fundamental frequency, so this value may be exaggerated.

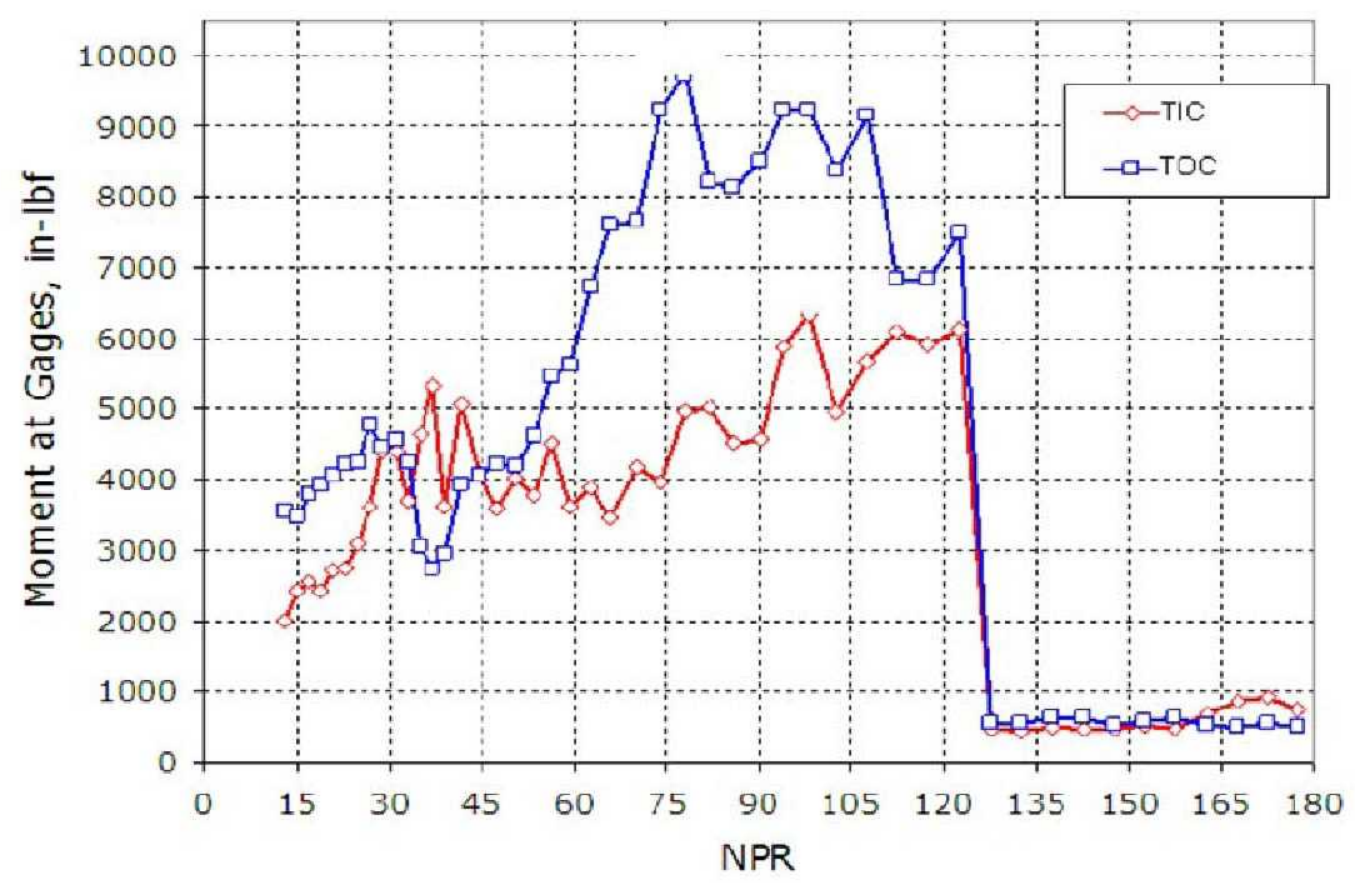

Figure 7. Strain-Gage Derived Excitation Moment for Two Nozzle Contours vs. NPR

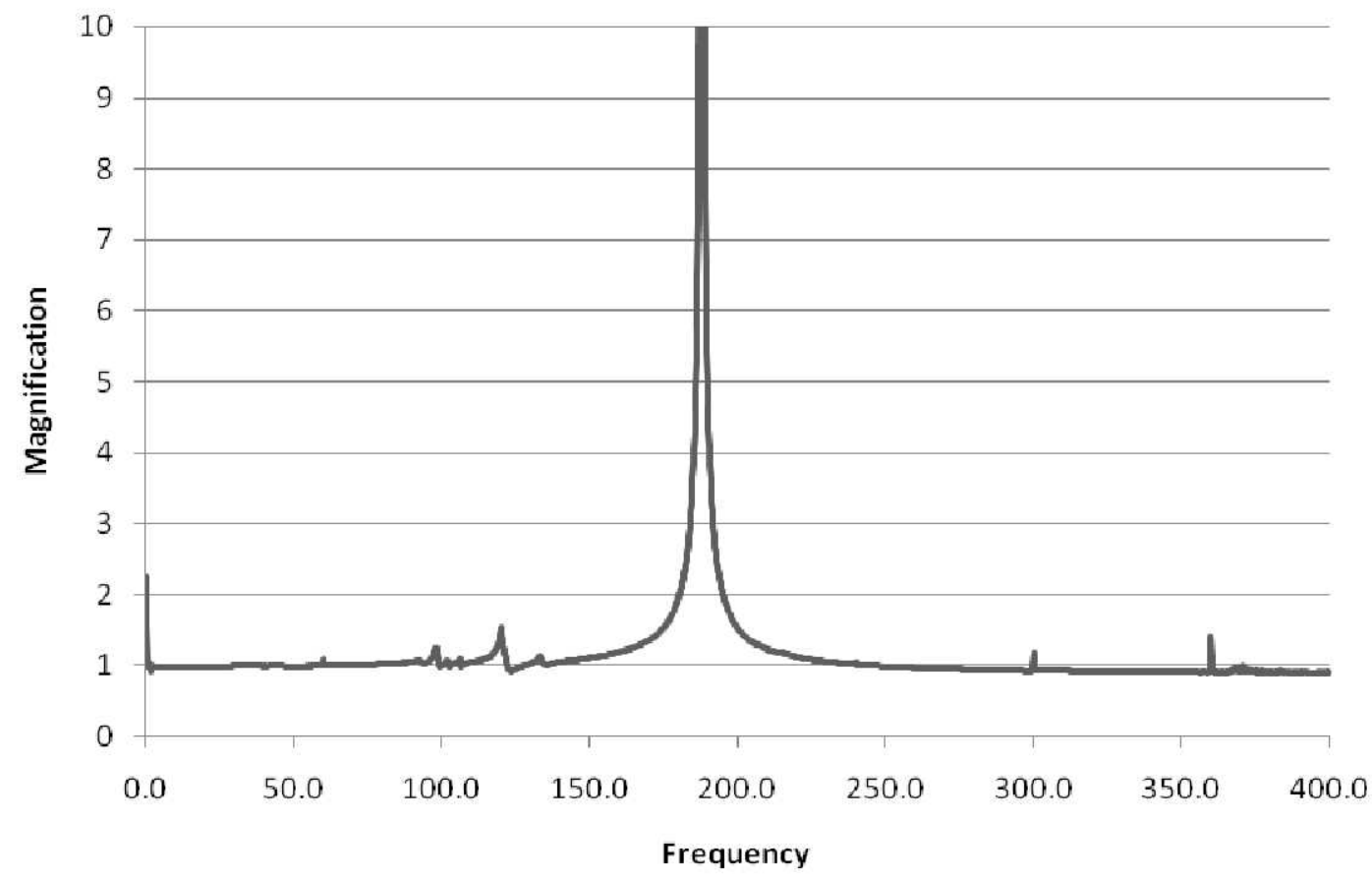

Figure 8. Dynamic Magnification of Measured Force

American Institute of Aeronautics and Astronautics 

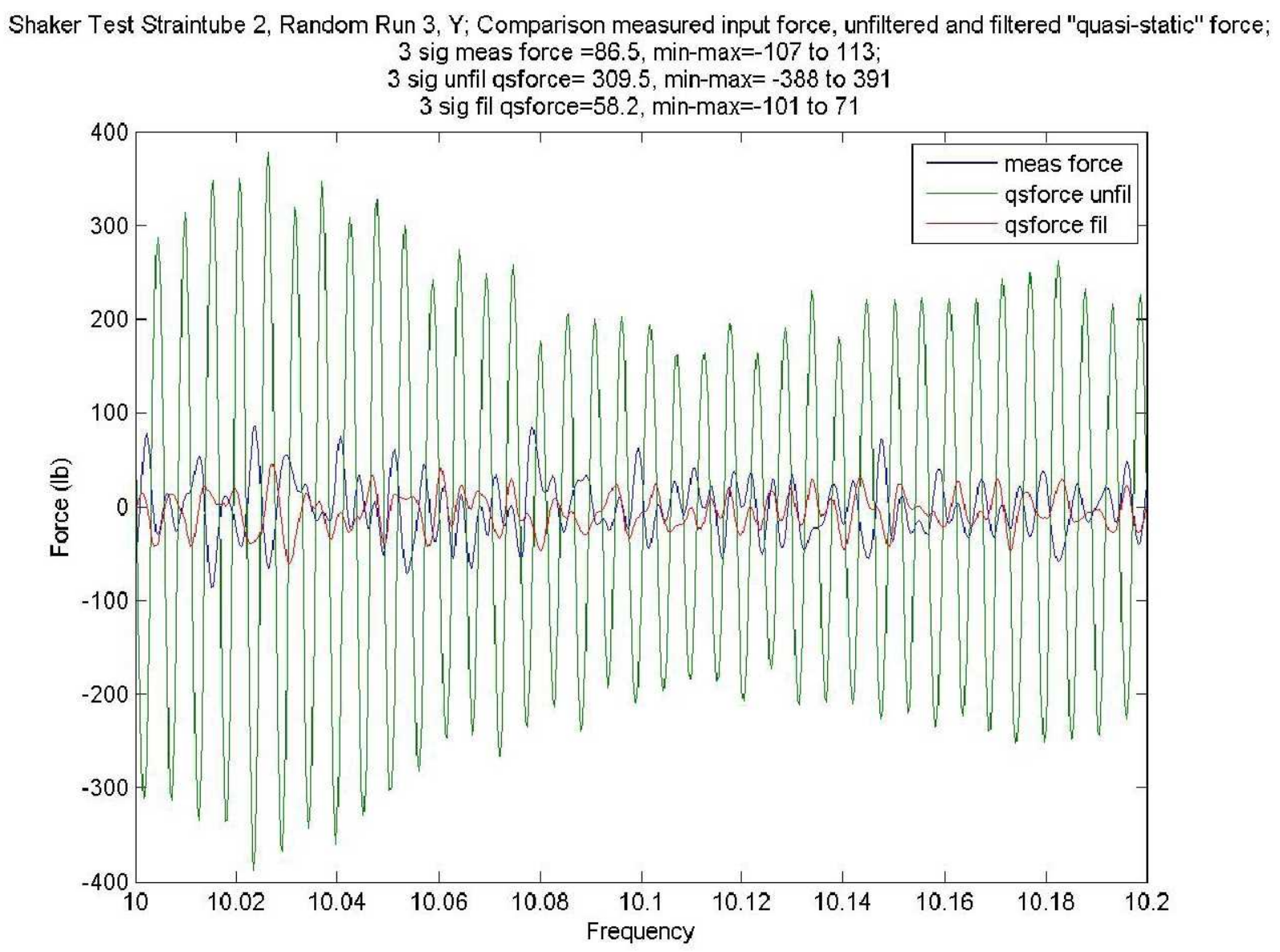

Figure 9. Comparison Measured Force, Filtered and Unfiltered Derived Forces

\section{Examination of Spectral Energy}

Since the aerodynamic excitation is of a different spectral character than the shaker test, it now becomes evident that the filtered strain gage derived force may be a reasonable approximation of the aerodynamic side load itself. This hypothesis was examined by a detailed study of the energy content of the side load tests. First, a subset of the runs for each of the two nozzle contours was selected based upon the clarity of the onset of the types of flow separation characteristics. The specific Kulite high frequency pressure gages that were located at the flow separation location and the time in the test at which these separations occurred were then identified. The PSDs of these gages at these time were then examined and a bounding low value $\mathrm{x}$ chosen to exclude spurious very low frequency measurements that might not be real (usually between 3 and $7 \mathrm{~Hz}$ ). The root mean square (RMS) values were then calculated for $\mathrm{x}-300 \mathrm{~Hz}$ and for $\mathrm{x}-166 \mathrm{~Hz}$, and the differences calculated (see Table 1). The average difference for the non-trivial loadings (less than $0.01 \mathrm{psi}$ RMS) is $7.2 \%$, implying that filtering to the unmagnified portion of the frequency range of the excitation only removes this amount of measured energy (the first case, which has the most decrease, has magnitudes an order of magnitude less than the others, so perhaps it should be discounted). The authors believe these errors are low enough to allow the use of the "quasi-static" strain-gage derived excitation force as an engineering estimation of the actual dynamic excitation force. 
Table 1. Comparison of Pressure Spectral Energy in Unfiltered and Filtered Ranges

\begin{tabular}{|c|c|c|c|}
$\begin{array}{c}\text { Contour } \\
\text { type }\end{array}$ & $\begin{array}{c}\text { NPR with highest } \\
\text { response }\end{array}$ & $\begin{array}{c}\text { Number of Kulites } \\
\text { with significant } \\
\text { response }\end{array}$ & $\begin{array}{c}\text { Average \% decrease in } \\
\text { RMS from x-300 Hz } \\
\text { to x-166 Hz }\end{array}$ \\
\hline TIC & 37 & 5 & $18 \%$ \\
\hline TIC & 41.5 & 5 & $2 \%$ \\
\hline TIC & 98 & 4 & $3 \%$ \\
\hline TOC & 31 & 5 & $6 \%$ \\
\hline TOC & 74 & 4 & $5 \%$ \\
\hline
\end{tabular}

\section{Conclusion and Future Work}

A long-term effort to characterize the transverse "side loads" experienced by rocket engines during start-up and shutdown transients has been undertaken at NASA/MSFC. The thrust of the most recent effort has been to provide estimates of the maximum magnitude of the load for use in the design of the new J2-X engine. This paper describes a procedure that uses statically-calibrated strain gage measurements to generate the magnitude of the forcing function by filtering out the portion of the signal that is mechanically amplified by the structural dynamics of the sub-scale nozzle and its test fixture. This value can be used for a quantitative comparison between the side loads for the TIC nozzle and the standard TOC nozzle. In addition, the value is shown to be within $7 \%$ of the actual magnitude of the forcing function. This fact will enable meaningful comparison with the existing baseline method for calculating the side load magnitude, which is the "Skewed Shock Plane Method," and with CFD calculations. A future paper by the authors will detail the methodology used for providing the rigorous "knockdown factor" between the two types of nozzle contours, which includes corrections for facility effects as well as a thorough evaluation of uncertainties. In addition, the future paper will further characterize the aerodynamic forcing function, including evaluation of low frequency highly directional components of the force.

\section{References}

\footnotetext{
${ }^{1}$ Ostlund, Jan, Supersonic Flow Separation with Application to Rocket Engine Nozzles, Doctoral Thesis, Royal Institute of Technology, Stockholm, Sweden, 2004

${ }^{2}$ Brown, Andrew M., Ruf, Joe, Reed, Darren, D’Agostino, Mark and Keanini, Russell, "Characterization Of Side Load Phenomena Using Measurement of Fluid/Structure Interaction," AIAA 2002-3999, 2002

${ }^{3}$ Smalley, K., Brown, A.M, Ruf, J, Gilbert, J., Flow Separation Side Loads Excitation of Rocket Nozzle FEM, AIAA 20072242, 48'th AIAA Structures, Structural Dynamics, and Materials Conference, Honolulu, Hawaii, April 23-26, 2007

${ }^{4}$ Nave, L. H., Coffey, G. A., Sea Level Side Loads in High-Area-Ratio Rocket Engine," AIAA 73-1284, Nov. 1973

${ }^{5}$ Frey, M, et. al, Subscale Nozzle Testing at the P6.2 Test Stand, 36 ${ }^{\text {th }}$ AIAA Joint Propulsion Conference, 17-19 July 2000, Huntsville, Alabama

${ }^{6}$ Brown, A.M., Ruf, J. Calculating Nozzle Side Loads using Acceleration Measurements of Test-Based Models, 25'th International Modal Analysis Conference (IMAC XXV), Orlando, Fl, Feb. 19-22, 2007

7 Kammer, Daniel C., "Input Force Reconstruction using a Time Domain Technique," AIAA Dynamics Specialists Conference, Salt Lake City, UT, Apr. 18, 19, 1996, Technical Papers (A96-27111 06-39), Reston, VA, American Institute of Aeronautics and Astronautics, 1996, p. 21-30
} 